\title{
Basal cell markers:34BE12 and p63, improving detection of basal cells in atypical prostatic lesions
}

Inas A. Rasheed*, Alaa Ghani Hussein**

\section{ABSTRACT}

Background: The diagnosis of prostatic pathology may be of challenging, as some difficult and suspected, atypical cases may lack basal cell layer by routine H\&E sections . Antibodies against 34BE12(HMW-CK) and p63 aid the diagnosis of such cases, to distinguish benign from malignant prostatic lesions.

Objective: to identify basal cells in atypical prostatic lesions ,and distinguish benign from malignant prostatic lesions.

Type of the study: A retro-spective study.

Methods: 115cases of paraffin embedded prostatic tissue blocks, diagnosed as : 76 cases were benign prostatic hyperplasia( $\mathrm{BPH}), 9$ cases were high grade -prostatic intraepithelial neoplasia (HG-PIN), and 30 cases were prostatic carcinoma(PCa) .Sections from each blocks were prepared for immunostaining with 34BE12 and p63.

Results : basal cells were detected in cases of $\mathrm{BPH}$, and HG-PIN , and absent in all cases of prostatic carcinoma ,using basal cell markers. Negative benign glands(>2) were found in $71.6 \%$ and $38.2 \%$ for $\mathrm{BPH}$ and $57.1 \%$ and $55.6 \%$ for HG-PIN immunostained with high molecular weight cytokeratin (34BE12) and $\mathrm{p} 63$, respectively, and significantly reduced to $9.0 \%$ and $11.1 \%$ for $\mathrm{BPH}$ and HG-
PIN, respectively with combined using of both markers .Conclusion : Combination of both basal cell markers (34BE12, p63) improving basal cell detection in atypical ,suspected prostatic lesions and distinguish benign from malignant lesions.

Key words: BPH ,HG-PIN , prostatic carcinoma , basal cell markers, 34BE12, HMW-CK, p63.

\section{Al-Kindy College Medical Journal 2017: Vol. 13 No.2 Page: 56-58}

${ }^{*}$ M.B.Ch. B., M.Sc.(Path), department of pathology , college of medicine , Al-Nahrain University .

${ }^{* *}$ M.B.Ch.B., M.Sc.(Path), F.I.C.M.S.(Path) ,professor of pathology, department of pathology, college of medicine , Al-Nahrain University..

Received $7^{\text {th }}$ Sept 2016, accepted in final $22^{\text {th }}$ May 2017 Corresponding to: Inas A. Rasheed. Iraq , Baghdad. Department of pathology , college of medicine , Al-Nahrain University
T he diagnosis of prostatic carcinoma on routine $\mathrm{H} \& \mathrm{E}$ sections may be challenging, as some atypical, suspected lesions may lack basal cells $(1,2,3)$. Basal cell layer well preserved in benign prostatic lesions, but absent and distorted in prostatic carcinoma $(3,4,5)$. Antibodies against 34BE12 and p63 aid the diagnosis, and distinguish benign from malignant lesions $(6,7)$. Technical factors e.g. formalin fixation and antigen retrival may affect the results and make difficult detection of basal cells stained with either 34BE12 and p63. Therefore, combination of both markers (34BE12 and p63), improving basal cell detection in benign prostatic lesions $(7,8,9,10)$.

Aim of the study : is to identify basal cells in atypical prostatic lesion on H\&E sections, by immunohistochemical markers ( 34BE12 and p63), and to distinguish benign from malignant prostatic lesions.

Material and methods : This a retro-spective study was approved by Institute Review Board of College of Medicine IAl-Nahrain University . The period of sample collection last from March /2015 to February 2016 , a total of 115 cases of formalin fixed paraffin embedded prostatic tissue of which (76) cases were BPH , (9) cases were HG-PIN , and (30) cases were prostatic adenocarcinoma .The paraffin blocks were retrieved from the histopathology archive of teaching laboratory in Medical City, for the period from 2013 to February 2016.For each case. One representative (4 micrometer) section was stained with Hematoxylin and Eosin, and histopathological diagnosis was revised, other two (4 micrometer ) sections were placed on positively charged slides and stained immunohistochemically using three steps -indirect streptavidin method for monoclonal mouse antibodies including anti -34BE12 antibody, and anti-p63 antibody, both manufactured by Dako .

Interpretation of the results of immunohistochemical staining :

1. HMW-CK (34BE12): brownish cytoplasmic staining of basal cells, with complete $(>75 \%)$, or partial $(\geq 25 \%)$ circumferential staining of the benign glands, is considered positive .

2.p63 : brownish nuclear staining of basal cells, with complete $(>75 \%)$, and partial $(\geq 25 \%)$ circumferential staining of benign glands, is considered positive .

The density of basal cell staining(for both $34 \mathrm{BE} 12$, and p63) were evaluated and scored as :1+(weak) ,2+(moderate), 3+ (strong). Basal cell staining was considered positive if moderate intense staining and $\geq 25 \%$ of the glands were stained. Number of negative benign glands were evaluated, and 2 negative benign glands were accepted as false negative.

Statistical analysis :was performed with SPSS V.16, using ttest with $p$ value of $<0.05$.

Results : All cases(100\%) of prostatic carcinoma were lack basal cell markers (both 34BE12, and p63).For cases of $\mathrm{BPH}, 97.3 \%$ and $100 \%$ showed 
immunoreactivity for 34BE12, and p63 respectively, with various pattern (complete and partial ) , and intensity ( moderate to strong ), fig(1). For BPH , negative benign glands $(>2)$ were found in $71.6 \%$ and $38.2 \%$ of cases stained with 34BE12 and p63, respectively, but significantly reduced to $9.0 \%$ when combination of both markers (34BE12 and $p 63)$ were used $(p=0.012)$, Fig(3). The difference in immunoreactivity that seen between both factors may be due to susceptibility of 34BE12 to formalin fixation. For HG-PIN , 77.8\% , and $100 \%$ of the cases showed basal cells immunoreactivity for 34BE12 and p63, respectively, with various pattern(complete and partial ), and intensity (moderate to strong), fig(2). Negative benign glands (>2) were found in $57.1 \%$ and $55.6 \%$ for 34BE12 and p63, no significant difference $(p=0.62)$ was found between them Combination of both markers (34BE12 and p63) to stain the same cases of HG-PIN significantly reduced the negative benign glands to $11.1 \%(p=0.37), \mathrm{Fig}(4)$

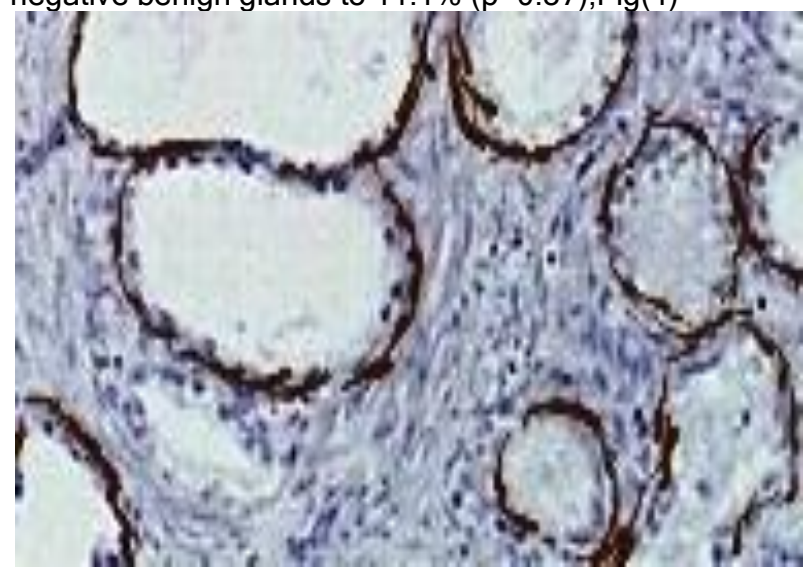

Fig(1). BPH with circumferential, brownish cytoplasmic , immunostaining of the basal cells of benign glands, 34BE12 , x10

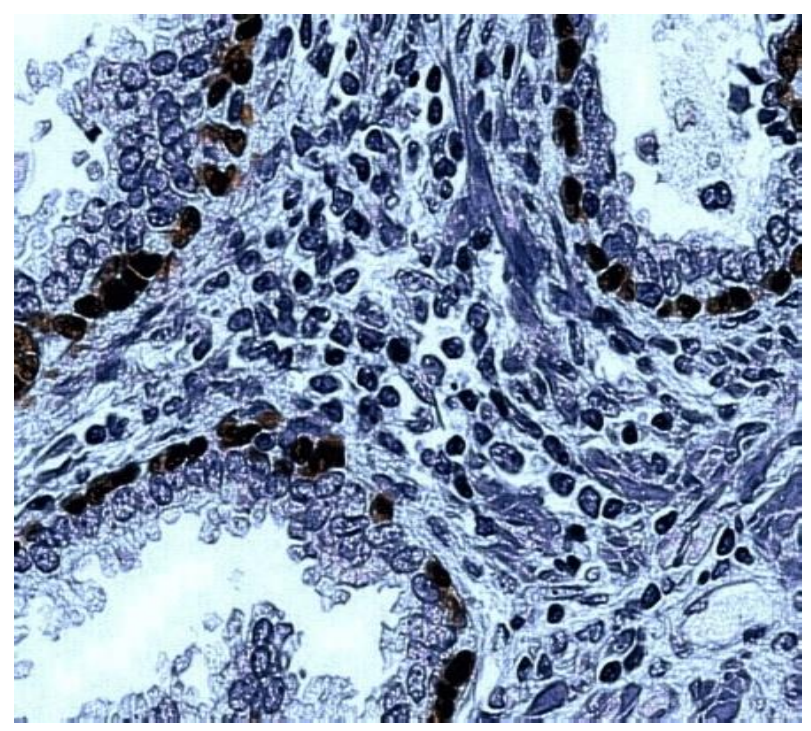

Fig(2) HG-PIN , show basal cell , brownish nuclear staining for $p 63, \times 20$

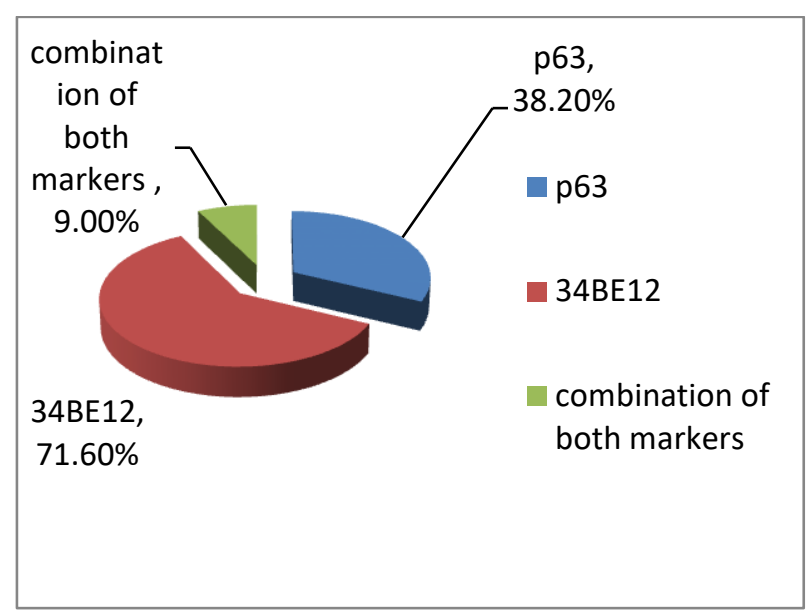

Fig(3). false negative benign glands(>2) lack basal cells staining for each marker (34BE12, and p63) and in combination of both markers in $\mathrm{BPH}$

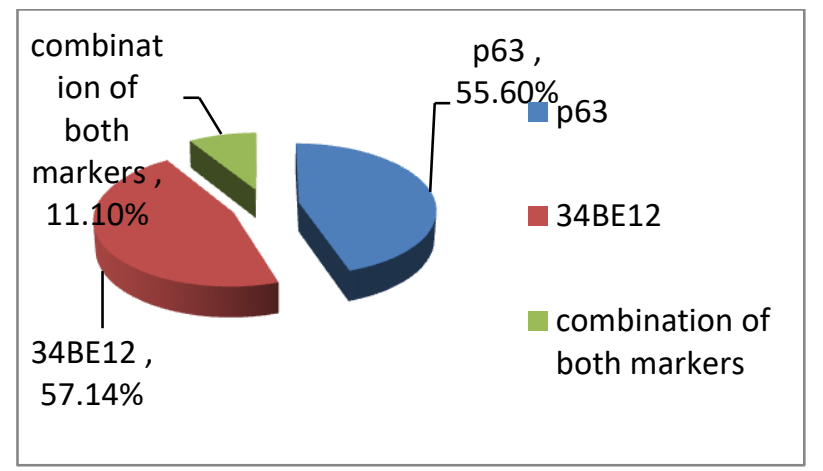

Fig(4). False negative benign glands( $>2$ ) lack basal cell staining for each marker(34BE12, and p63) and in combination of both makers in HPIN

Discussion : For current study , 74 cases (97.3\%) of BPH showed positive immunoreactivity of the basal cell for HMW-CK(34BE12), and all cases (100\%) were immunoreactive for p63. Negative basal cell immunostaining of benign glands (>2 glands) was found in $71.6 \%$ and $38.2 \%$ among presented cases of $\mathrm{BPH}$ for 34BE12 and p63, respectively. These negative glands lacking basal cell immunoreactivity, were significantly reduced to $9.0 \%$ when combination basal cell markers were used for the same cases .For current study , 77.8\% and $100 \%$ of cases of HG-PIN showed basal cell immunoreactivity for 34BE12and p63, respectively. No significant difference was found between complete and partial patterns for basal cells immunostaining . Lacking of basal cell immunoreactivity for $>2$ benign glands of presented cases of HG-PIN was found in $57.14 \%$ and $55.6 \%$ for $34 \mathrm{BE} 12$ and $\mathrm{p} 63$, respectively . Using combination of basal cell markers (i.e. 34BE12 and p63) dramatically reduce negative benign glands for the same cases of HG-PIN to $11.1 \%$. The difference in immunoreactivity that seen between both markers may be due to susceptibility of 34BE12 to formalin fixation

Regarding presented cases of PCa lack basal cell layer, and showed negative immunostaining for both HMW-CK and p63.Shah R etal in 2004 ,found variable basal cell 
staining intensity and pattern with HMW-CK(34BE12) and p63 for $\mathrm{BPH}$ and GH-PIN was found . Benign glands lack basal cell lining found in $2 \%, 6 \%$ and $2 \%$ of histologically benign glands with combination, 34BE12 and p63 staining, respectively. Combination of basal cell markers increase the sensitivity of basal cell detection (9)Montironi $R$ etal in 2006 , reported that combination of two basal cell markers (34BE12, and p63)increase the sensitivity of basal cell detection, compared with using either marker alone, and of value in the diagnosis of atypical prostatic lesions on H\&E sections .(10)

Shiran MS et al in 2007 , reported that p63 is a useful complementary basal cell stain to 34BE12, and helpful to pathologist in dealing with difficult prostatic lesions. Basal cell immunostaining for 34BE12 and p63 was found in $100 \%$ of the cases of BPH and HG-PIN . The number of benign glands $(>2)$ showing absence of basal cell staining for 34BE12 and p63 were small , accounting for $9.3 \%$ and $11.6 \%$, respectively.(10)Paner GP etal in 2008 , found that basal cell markers (p63, and 34BE12) useful in confirming whether prostatic glandular lesions are benign or malignant .(11)Zynger DL etal in 2009 , recommended the use of both basal cell markers (34BE12, and p63) in diagnosis and differentiation of atypical prostatic lesions from prostatic carcinoma (12)Dabir PD et al , in 2012 showed all cases of BPH and HG-PIN were immunoreactant for HMW-CK(34BE12) and p63, and the continuous pattern was the dominant for both markers (87.5\% of $\mathrm{BPH}, 14$ out of 16 ) and $100 \%$ for HG-PIN. All cases of $\mathrm{PCa}$ lack expression for both HMW-CK and p63.(13)Kalantari MR etal in 2014 , reported that combination of both basal cell markers (34BE12, and p63) is helpful for improving differentiation of prostatic carcinoma from cancer mimickers .(14) These studies support the findings of the current study that combination of both markers(34BE12 , and p63 ) improving the detection of basal cells in atypical prostatic lesions and distinguish the benign from malignant prostatic lesions .

Conclusion: We concluded that :

1. Basal cell markers ( 34BE12 and p63) of valuable help in distinguishing between benign and malignant prostatic lesions in atypical, difficult and suspected cases, which show challenging .

2. Combination of both basal cell markers (34BE12 and p63) improving basal cell detection .

Author contributions : All authors contributed to this manuscript .They coordinated study subject recruitment , implementation and progress of this study, and helped with data interpretation and manuscript organization and editing .

Conflict of interest : All authors have no conflict of interest .

Funding : This research was funded by College of Medicine IAI-Nahrain University .

Acknowledgment : Special thanks to all staff members of pathology department in Al-Nahrain University /College of Medicine for their valuable help and advice .

List of abbreviation :

-BPH : benign prostatic hyperplasia

-HG-PIN : high grade prostatic intraepithelial neoplasia -HMW-CK : high molecular weight cytokeratin .

-PCa: prostatic carcinoma .

\section{References:}

1- Bostwick D .Prospective origins of prostate carcinoma : PIN and atypical adenomatous hyperplasia .Cancer , 1996, 78: 330-35
2- Mwakyoma HA. The prevalence of high grade prostatic intraepithelial neoplasia in prostatic biopsy diagnosed as benign prostatic hyperplasia at Muhimibili National Hospital, Dar es Salaam. Tanzania Medical Journal (TMJ ).2008 , 23(1): 1-4.

3- Taneja SS , Morton R , Barnette G , Sieber P , Hancock ML , Steiner M . Prostate cancer diagnosis among men with isolated high grade intraepithelial neopalsia enrolled onto a three years prospective phase III clinical trail of oral toremifene .J Clin Oncol.2013, 3(5): 523-9

4- Al-Saadi H, Mahdi EM . Detection of prostate cancer using tumor markers and biopsy in outpatient in Kerbala city .Iraq.International Research Journal of Medical Sciences .2013, 7: 17 21

5- Gleason DF . Atypical hyperplasia , benign hyperplasia , and well differentiated adenocarcinoma of the prostate.Am J Surg Path , 1985, 9(3):53-66.

6- Kristiansen G, Epstein $\mathrm{JI}$. Immunohistochemistry in prostate pathology. Dako, Agilent Technologies company. 2008

7- Bostwick D, Qian J , Ramani D. Immunohistochemistry of the prostate and bladder , testis, and renal tumors.Diagnostic immunohistochemistry .Philiadelphia.2002, 407-34.

8- Shah RB , Zhou M, Leblanc M, Rubin MA. Comparison of the basal specific marker 34BE12 and p63 in the diagnosis of prostate cancer. Am J Surg Pathol. 2002, 26(9): 1162-8

9- Shah RB, Kunju LP, Shen R. Usefulness of basal cell cocktail (34betaE12 +p63) in the diagnosis of atypical prostate glandular proliferation. Am J Clin Pathol. $2004,122(4)$

10- Montironi R, Scattoni V, Mazzucchelli R . Atypical foci suspicious but not diagnostic of malignancy in prostatic needle biopsies .Eur Uro, 2006 , 50:666674.

11-Shiran MS, Tan GC , Sabariah AR , Rampal L , Phang KS .p63 as a complimentary basal cell specific marker to high molecular weight cytokeratin in distinguish prostatic carcinoma from benign prostatic lesions.Med J Malaysia. 2007, 62(1):36-9.

12- Paner GP , Luthringer DJ , Amin MB . Best practice in diagnostic immunohistochemistry : prostate canrcinoma and its mimics in needle core biopsies . Bio Med Search .2008

13-Zynger DL ,Yang $X$. High -gade prostatic intraepithelial neoplasia of prostate : the precursor lesion of prostate cancer . Int J Clin Pathol. 2009, 2:327338.

14- Dabir PD , Ottasen P . Comparative analysis of three and two -antibody cocktails to AMACR and basal cell markers for immunohistochemical diagnosis of prostate carcinoma . Diagnostic pathology.2012, 7:81.

15- Kalantari MR, Anvari K, Jabbari H, Varshoee tabrizi . p63 is more sensitive and specific than 34BE12 to differentiate adenocarcinoma of prostate from cancer mimickers. ljbms.2014, 17 :497-501. 